\title{
A PLAN FOR INDEXING CASES IN HOSPITALS FOR THE INSANE.
}

\author{
By WILliaM A. WHITE, M. D., \\ AND \\ FRANCIS M. BARNES, JR., M. D.
}

The keeping of the records of the symptomatology, treatment and course of a disease process dates far back to the time of Hippocrates, and it was Sydenham who advised the writing down in permanent form the records of our observations so that they might become of value to others in their task of alleviating the sufferings of the unfortunate sick. This applies both to the private practitioner and to the hospital, but it is especially with the latter that this paper has to deal. That such observations are being recorded will be strikingly evident to one who enters the splendidly equipped record rooms of some of our modern hospitals. The comparatively recent advent of modern filing devices has made the physical conditions almost ideal and the old, cumbersome case books are rapidly disappearing from the shelves.

In a large hospital where hundreds of patients are received annually and where thousands are continuously cared for, the keeping of suitable records is a matter of most definite importance. This is not only true of the general hospitals, but particularly of institutions intended primarily for the care and treatment of individuals suffering from mental disorders for the reason that the state of development of the psychiatric branch of medicine is yet in its infancy and anything which will tend to promote in any degree the progress of our knowledge of mental disorders is to be welcomed and encouraged. And in this regard, given the hospital and the patient, probably nothing will be of more value and importance in furthering our studies than sufficiently complete records of the cases observed.

Perhaps next to the importance of making the records is their preservation, not only in suitable, but accessible form. Most excellent records may be made but their existence serves but a transient and temporary use if they are not so stored that they are 
readily accessible. There is no doubt but that a great wealth of valuable clinical observations is being lost constantly because it is so thoroughly hidden among thousands of records that it cannot be found when wanted without expending an almost prohibitive amount of labor in the search.

Suppose, for instance, one wishes to look through the records of the hospital for a certain group of cases, let us imagine for the moment that these are desired for statistical purposes. How are all of these case histories to be obtained with the least expenditure of effort and time? Supposing one wishes to investigate the comparative frequency of vascular degenerative processes in the deteriorating psychoses of adolescence and in manic-depressive psychosis from both the clinical and pathologic standpoint. It becomes necessary to have some relatively simple and expeditious means for making these case histories accessible. If one is obliged to look through the histories of several thousand cases to obtain those desired, this amount of clerical labor in itself would often offer sufficient reason for abandoning the project. In other words, a vast amount of material is never worked up simply because of the physical difficulties which arise, largely owing to the fact that the mere abundance of material, which in itself is of value from the statistical standpoint, renders it inaccessible. On this account it is evident that if there was some simple means at hand by which the material could be gathered together greater use could be made of otherwise wasted observations. The following plan, now in use in this hospital, seems to offer a fair solution to the question and it is with this view in mind that it is here presented with the hope that it may prove of service to others who have similar problems with which to deal.

Although spoken of as a case catalogue there are in reality three separate catalogues cross indexed with each other. These are arranged according to the diagnosis. First, there is the catalogue for the mental diagnoses as made clinically; second, the clinically diagnosticated physical condition; and third, the pathologic findings. For simplicity these are designated by the numbers I, II and III, and there are three sets of unruled cards, each card of each set bearing its respective number in the upper left-hand corner. Nothing else is printed on the cards. A three- by fiveinch card affords ample space for all data required. To illustrate 
I9II] WILliaM A. WHITE AND FRANCIS M. BARNES, JR. 599

this let us take a case of dementia precox of the hebephrenic type in which cardio-vascular lesions had appeared to influence the mental symptoms, and in which the autopsy showed the presence of a cerebral tumor. The card for the mental diagnosis in this case would read as follows:

I. Dementia precox, hebephrenia.

Brown, John W. (Col.) Dc. 1874I. Cl. Vol. 206.

II. Cardio-vascular disease.

III. Tumor, cerebral, cardio-vascular disease.

This is card I bearing at the top the mental diagnosis. Below this comes the patient's name. The abbreviation Col. indicates colored and the Dc. following this indicates that the patient is dead. Likewise Ds. would indicate discharged. Then comes the case number followed by the clinical volume in which this case history and complete records will be found filed. These points, of course, have a value only for this hospital where this method of filing is employed and might not fit other conditions without some slight modification. Card II, with the clinically diagnosticated physical condition, will be similarly arranged, the data will be entirely the same only there will be a transposition of the clinical diagnosis, cardio-vascular disease, and the mental diagnosis, hebephrenia.

II. Cardio-vascular disease.

Brown, John W. (Col.) Dc. 18741. Cl. Vol. 206.

I. Dementia precox, hebephrenia.

III. Tumor, cerebral, cardio-vascular disease. 
Card III will likewise be similar to Card I with the transposition of the pathologic diagnosis and the mental diagnosis.

III. Tumor, cerebral.

Brown, John W. (Col.) Dc. 1874I. Cl. Vol. 206.

I. Dementia precox, hebephrenia.

II. Cardio-vascular disease.

A second Card III will be needed for the diagnosis cardio-vascular disease.

Now with these three cards filed in separate correspondingly labeled compartments under their proper headings it becomes a simple task to gain access to all cases of dementia precox of the hebephrenic type, the pathologic findings that may have been associated with this mental disease, etc. In cases where there is no autopsy or where there has been no clinical diagnosis the corresponding cards are not used. Of course, the cataloguing is not always so simple a proposition. For instance, take a case of arteriosclerotic dementia in which there has been a physical diagnosis of cardio-vascular disease made, and in which, at autopsy, cerebral arteriosclerosis was discovered. Under these conditions the index would be simple enough. However, we may imagine that there was no autopsy performed and we may be left in doubt as to whether it is a simple senile dementia or an arteriosclerotic. In such an instance two I cards must be made out, in other words, we catalogue this case as to the mental condition under two headings, cross index it. And again, in this case there may have been a cerebral hemorrhage recognized clinically and then there must be two II cards, a second one for the cerebral hemorrhage. Still, again, this possible hemorrhage might not have been immediately fatal and it might become necessary to make a third II card for the hemiplegia which might have remained.

There is, it is recognized, a wide limit to the number of diagnoses that might be made in a given case, but a too detailed cata- 
19II] WILLIAM A. WHIte AND FRANCIS M. BARNES, JR. 601

loguing and cross indexing would in itself defeat the prime object of the scheme-simplicity and consequently, increased accessibility. On this account it is often preferable to use a group diagnosis, such as cardio-vascular disease, instead of detailing the separate lesions such as aortic obstruction, arterial degeneration, etc. For the same reason a case of general paralysis with autopsy will be entered on all three cards alike under the one heading, paresis. The various focal neurologic signs and the different lesions found at autopsy are known to be part and parcel of this one disease entity and are, therefore, disregarded in the index, although they may be found in the case record. The principle is that the term used in each instance, while being as specific as is consistent with the facts, shall be broad enough to surely include the condition. A condition of depression, for example, might be impossible of certain diagnosis. If it is catalogued as an undifferentiated depression it will be accessible for one who is studying involutional melancholia, manic-depressive psychosis and constitutional or psychopathic depression. Had it been classed as a symptomatic depression it would not have been included in the cases studied for any of the other conditions. The diagnosis, then, while being as specific as consistent with the facts must be broad enough to surely include the condition.

On appropriate guide cards used according to modern filing methods more specific cross indexing will be employed to facilitate the use of the catalogue by different individuals. These cards will bear more detailed information and will direct the user of the catalogue to the main group heading under which the condition for which he is searching will be found. For example, one looking for renal or cardiac diseases will be directed by these guide cards to look for these lesions in the group, cardio-renal disease, etc. Vice versa, as in the case of dementia precox which was above used as an illustration, under dementia precox will be found a "see also" card referring to hebephrenia, catatonia, etc. In other words, it is intended that anyone not experienced with the catalogue will be able to find quickly the cases for which he may be looking. It has also been deemed advisable to use the group diagnosis, the more comprehensive term, where possible, not only in order to prevent confusion due to excessive size of the catalogue, but for the reason that psychiatric nomenclature at the present day cannot 
be considered to be in a stable state and certain terms now used to designate particular mental conditions may be obsolete a few years hence. Such changes, should they occur, might be expected to effect the forms or phases which go to make up the groups. Therefore such terms are avoided as far as is consistent with sufficient clearness.

Another feature wherein the catalogue may seem to fall short on casual examination is that many of the conditions recognized clinically and at the autopsy table are not included. However, the index is used primarily from the psychiatric standpoint, so on this account only such conditions as are considered to bear on the mental disease are taken into consideration. For instance, it is of no immediate psychiatric importance that a senile dement happens to fall and sustain a fracture of the neck of the femur which ultimately hastens his death nor, likewise, is it of value to know that a much deteriorated hebephrenic dies of pneumonia. Therefore these physical accidents are not catalogued. On the other hand in the case of delirium associated with typhoid fever the latter becomes of importance as also does the renal condition in a case of uremia with manifestations of mental disturbance.

In many cases it is often difficult, even impossible, to make a clear diagnosis of the mental state. This has previously been mentioned. In many such instances we have resort to the heading, unclassified or undiagnosticated. In many of these cases where it has been impossible to arrive at a satisfactory diagnosis we have recourse to the undifferentiated symptomatologic diagnosis, a depression or an excitement, unclassified. However, it is often true that these cases are so diagnosticated because circumstances have prevented a thorough study of the case and so there is not so much loss, because in looking up a group of cases for study one would not be interested in those in which full and carefully completed observations had not been made.

Although it is possible that this method of cataloguing cases might be made of value as a means of keeping track of cases while still resident in the hospital, the amount of work which it would require to keep in touch with the changing diagnoses would make it almost impractical. It seems that any value derived from such a procedure would not only be slight, but also the information gained would be subject to repeated changes from time to 
IgII] WILliam A. WHITE AND FRANCIS M. BARNES, JR. 603

time. As intended to be used and as employed in this hospital, no case is entered until the individual's hospital career has been terminated, either by death or discharge. The diagnoses, as entered on the cards, are obtained as follows: When a patient is ready for discharge the history is presented in full at the staff conference, the records summarized and reviewed, the patient is presented and then diagnoses are made. Deaths, when autopsies have been held, are not presented until the report of the pathologist is submitted together with the other records in the case. In this way only final opinions, based on all of the evidence obtainable, eventually reach the catalogue where they are entered under the supervision of a member of the medical staff. In this way is obtained as valuable a permanent form as is possible.

It is not expected that this scheme is going to serve to pick out just exactly the variety of cases that may be desired in every instance, some work will still remain for the individual. Nevertheless, it will make it possible to obtain very easily and quickly all cases in the record room which have any relationship to the group sought after and then it becomes a comparatively simple matter to discard rapidly such cases as do not seem to be desired for the particular study. 